\title{
The Mobile Territorial Lab: a multilayered and dynamic view on parents' daily lives
}

Simone Centellegher ${ }^{1,4}$, Marco De Nadai ${ }^{1,4}$, Michele Caraviello², Chiara Leonardi' ${ }^{1}$ Michele Vescovi², Yusi Ramadian², Nuria Oliver ${ }^{5}$, Fabio Pianesi ${ }^{1}$, Alex Pentland ${ }^{3}$, Fabrizio Antonelli ${ }^{2}$ and Bruno Lepri1*

"Correspondence: lepri@fbk.eu ${ }^{1}$ Foundation Bruno Kessler, via Sommarive, 18, Trento, 38123, Italy Full list of author information is available at the end of the article

\section{算 Springer}

\begin{abstract}
The exploration of people's everyday life has long been of interest to social scientists. Recent years have witnessed a growing interest in analyzing human behavioral data generated by technology (e.g. mobile phones). To date, a few large-scale studies have been designed to measure human behaviors and interactions using multiple sources of data. A common characteristic of these studies is the population under investigation: students having similar daily routines and needs. This choice constraints the range of behaviors, of places and the generalization of the results. In order to widen this line of studies, we focus on a different target group: parents with young children aged 0 through 10 years. Children influence multiple aspects of their parents' lives, from the satisfaction of basic human needs and the fulfillment of social roles to their financial status and sleep quality.

In this paper, we describe the Mobile Territorial Lab (MTL) project, a longitudinal living lab which has been sensing by means of technology (mobile phones) the lives of more than 100 parents in different areas of the Trentino region in Northern Italy. We present the preliminary results after two years of experimentation of, to the best of our knowledge, the most complete picture of parents' daily lives. Through the collection and analysis of the collected data, we created a multi-layered view of the participants' lives, tracking social interactions, mobility routines, spending patterns, and personality characteristics.

Overall, our results prove the relevance of living lab approaches to measure human behaviors and interactions, which can pave the way to new studies exploiting a richer number of behavioral indicators. Moreover, we believe that the proposed methodology and the collected data could be very valuable for researchers from different disciplines such as social psychology, sociology, computer science, economy, etc., which are interested in understanding human behaviour.
\end{abstract}

Keywords: parents' daily life; mobile phone data; spending data; personality; computational social science

\section{Introduction}

The exploration of everyday life and mundane behaviors of people have long been of interest to social scientists $[1,2]$. Ethnographic methods - e.g. qualitative techniques for collecting insights on social life such as direct observation, interviews, diaries, and other fieldwork [3] - have been applied for studying social and cultural phenomena such as lifestyles [2], mobility [4], communication patterns [5], cultural specificities [6], and so on

(c) 2016 Centellegher et al. This article is distributed under the terms of the Creative Commons Attribution 4.0 International License (http://creativecommons.org/licenses/by/4.0/), which permits unrestricted use, distribution, and reproduction in any medium, provided you give appropriate credit to the original author(s) and the source, provide a link to the Creative Commons license, and indicate if changes were made. 
[7]. While several benefits of applying such methods exist, a number of constraints make them scarcely applicable for large-scale studies: they are time-consuming, sometimes too specific to be replicated or applied generally, and they suffer from researcher bias, which makes them less appealing than quantitative approaches [7].

Recent years have witnessed a growing interest toward the usage of personal data coming from the behavioral traces left by people using technologies. In particular, the almost universal adoption of mobile phones is generating an enormous amount of data about human behaviors with a breadth and depth that was previously inconceivable $[8,9]$. These devices are able to sense (i) location, (ii) other devices in physical proximity through Bluetooth scanning, (iii) communication data, including both metadata (logs of who, when, and duration) of phone calls and text messages (SMS), etc. Correspondingly, the availability of huge streams of personal data related to activities, routines and social interactions is continuously growing [10-13], which represents a novel opportunity to challenge and often subvert long-lasting paradigms in social sciences $[14,15]$ and to address fundamental problems of our societies in different fields, such as mobility [16-18], finance [11, 19], personality and emotions [20-22] and healthy living and subjective well-being [23-27].

Recently, some large-scale studies were designed to measure human behaviors and interactions using multiple sources of data. In the Reality Mining study, data from 100 mobile phones of business school students were collected over a nine-month period [28]. In the Social Evolution experiment $[12,23]$, the everyday life of a whole undergraduate dormitory was tracked for almost 8 months using mobile phones (call logs, location, and proximity interactions). This experiment was specifically designed to study the adoption of political opinions, the spreading of epidemics, the effect of social interactions on depression and stress, and the eating and physical exercise habits. In the Friends and Family study, 130 graduate students and their partners, sharing the same dormitory, carried smartphones running a mobile sensing platform for 15 months [11]. Additional data were also collected from Facebook, credit card statements, surveys including questions about personality traits, daily mood and sleep quality, group affiliations, etc. The Student Life study [26] used Android phones to collect the day-to-day and week-by-week impact of workload on stress, sleep, activity, mood, sociability, mental well-being and academic performance of a single class of 48 students across a 10 week term at Dartmouth College. Currently, the Copenhagen Networks Study [13] is collecting data on face-to-face interactions, mobile phone communications, Facebook usage, location, demographics and other individual characteristics for a population of almost 1000 students.

A common characteristic of the previous studies is the population under investigation: undergraduate or graduate students having similar daily routines and needs (e.g. hanging out at the same places, sleeping in the same dormitory, etc.). This choice limits the range of behaviors (e.g. studying, attending classes, hanging out with friends, doing sport, etc.) and the range of places (e.g. cafeteria, canteen, library, etc.). In order to contribute to widen this line of studies, we focus on a different target group: parents with young children aged 0 through 10 years. The age range was set to include infancy to middle childhood, because in this phase parents' life is driven by children needs more than their own [29]. Children are the fount of our greatest joys and the source of our greatest worries. Many parents consider raising children to be one of the most gratifying [30] - but also one of the most stressful and challenging [31] - life experience. Indeed, children influence multiple aspects of their parents' lives, from the satisfaction of basic human needs and the fulfillment of social roles 
to their influence on financial status and sleep quality. The after-work time of parents is mostly spent with children. This means that parents' routines and social interactions are highly determined by children needs and this fact often turns leisure activities to a mix of leisure and family duties [32]. Similarly, parenthood affects the financial situation and the spending behavior: parents typically make multiple financial sacrifices paying for their children's food, clothing, medical care, and schooling [33]. For this reason, the association between parenthood and well-being and the analysis of daily life of parents have become hot topics among sociologists, psychologists, and economists (for a general review, see [34]).

In this paper, we describe a longitudinal study, the Mobile Territorial Lab (MTL) living lab study, that has been observing the lives of more than 100 parents through multiple channels for more than two years. We have been collecting data from multiple sources, including smartphones, questionnaires, experience sampling probes, etc. Data from all of these sources have been used to create a multi-layered view of the lives of the study participants. In particular, we have been tracking social interactions (e.g. call and SMS communications), mobility routines and spending patterns, etc. Additionally, we have conducted some interventions to explore the daily monetary valuations of four categories of mobile personal data (phone communications, app usage, location data and media usage) [35] and the location-sharing preferences of the participants [36]. In the following sections we describe the experimental plan and the data collection methodology. Then, we report initial results from the MTL project, primarily in the form of an overview of the collected data and of an initial analysis of the participants' communication, mobility, spending behaviors and their interactions with personality traits and life satisfaction. We hope the work presented here will serve as a guideline in the design of similar in-the-wild studies.

\section{Methods: data collection}

In this section we describe the recruitment process, the socio-demographic characteristics of our study participants, the data collection platform, the multiple sources of data we collected (mobile phone data, spending data, questionnaires), and the personal data management tool provided to our study participants in order to control and share with each other the collected data.

\subsection{Target group and community management}

The Mobile Territorial Lab (MTL) consists of a group of 142 volunteers carrying an instrumented smartphone in exchange for a monthly credit bonus of voice, SMS and data access. All volunteers were recruited within the target group of young families with children, using a snowball sampling approach, where existing study subjects recruit future subjects from among their acquaintances [37]. Upon joining the living lab, each participant filled out several questions to collect socio-demographic information (e.g. age, gender, number of children, personal and familiar net income). The participants' age ranges from 28 to 50, with an average of 39.19 (std. dev. of 3.94). 90 participants are women (63.38\%) and 52 are men $(36.62 \%) .138$ participants are Italian $(97.18 \%)$ and 4 participants are from other countries $(2.82 \%)$. They have a variety of occupations and education levels, ranging from high school diplomas to $\mathrm{PhD}$ degrees. 41 participants have one child, 76 have two children and 25 have three children. Figure 1 shows the distributions of the participants' personal and familiar incomes respectively. All the participants are savvy Android users. 


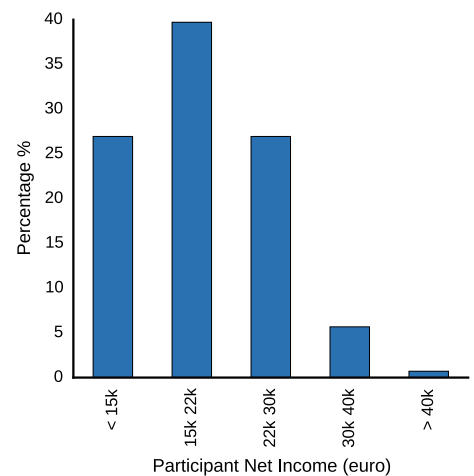

a)

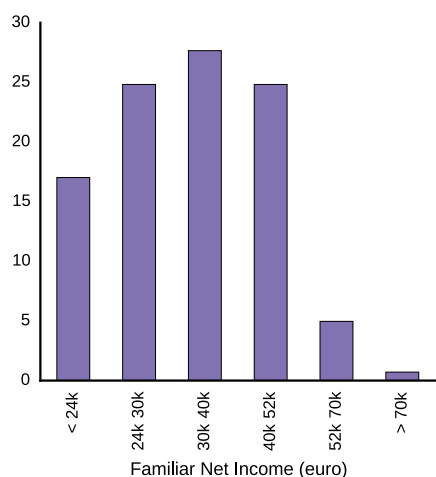

b)

Figure 1 Personal and family income levels distributions. Histograms of the distributions of (a) participants net income levels and (b) familiar net income levels of the Mobile Territorial Lab community.

Upon agreeing to the terms of participation, the volunteers granted researchers legal access to their behavioral data collected by their smartphones.

Participants retain full rights over their data such that they can order deletion of personal information from the secure servers where it is stored. Moreover, they have the choice to participate or not in a given study. Following the Italian regulations, all participants were asked to sign informed consent forms and the study was conducted in accordance to them. The form and the MTL study were also approved by a joint Ethical Committee of University of Trento and Province of Trento. In order to keep participants engaged throughout the project life-cycle, community management strategies have been employed. For example, several social events have been organized to communicate project objectives and tasks. Additionally, some applications have been provided as rewards, e.g. an application for helping families managing their free time. Community management is carried out by a public organization, called Smart Crowds [38], which supports research institutions and companies in setting up and coordinating living lab projects.

\subsection{Data collection platform}

Study participants were supplied with an Android phone equipped with a dedicated platform, based on a modified version of the open source framework FunF [11]. This platform periodically collects more than 25 different types of data signals with the goal of minimizing battery drain. In particular, it keeps track of the communications through call and SMS logs, and the user's location thanks to traditional sensors such as GPS and the location of the cell towers that the phone is connected to in the GSM network.

Additionally, the phone's platform includes a survey application which is able to periodically ask the participants some questions designed by the researchers in the context of a specific user study. Finally, the platform features a dashboard tool that allows the researchers and the community managers to control and monitor the progress of data collection and reporting critical issues. The dashboard enables the remote activation and de-activation of the different data sources, according to the ongoing research tasks. Furthermore it enables the setting of specific collection parameters such as the frequency of sampling.

The phone's data is locally saved in a 'delay tolerant' way: in the absence of network access, the phone accumulates the collected database files locally. When server connection is 
made (for example a participant connects to WiFi to browse the web), the system attempts to upload files to the data-store server.

\subsection{Data sources}

The data collected were obtained from the mobile sensing platform, using questionnaires, and from an ad-hoc experience sampling application for collecting daily expenses. In this section we describe in more detail the type of data we collected.

\subsubsection{Mobile phone data}

Several works have shown that mobile phone data is useful to study human mobility patterns and social interactions (see Blondel et al. [39] for a comprehensive review of recent advances in studies using mobile phone datasets). Thus, we collected call and SMS logs and location data from each participant's phone.

For each call log we kept track of the caller, the callee, the type of the call (ingoing, outgoing or missed), the timestamp of when the call was made and its duration. In the case of SMS logs we kept track of the sender, the recipient, the type of the SMS (inbox messages, sent messages) and the timestamp. In both kinds of logs the phone number of the contacted person is one-way hashed. Moreover, no conversational or textual contents were recorded.

For each location point we kept track of the user ID, the latitude, the longitude, the accuracy, the timestamp and the location provider which can be the Global Positioning System (GPS), the GSM network or the Fused Location Provider. The latter is an Android's location service, which combines cellular, WiFi, GPS, and sensor data to achieve balance between battery consumption and the accuracy of the geographical position. The estimated cumulative distributions function of the accuracy of collected location points are represented in Figure 2. As expected the GPS data is the most accurate sensor, with $82 \%$ of positions with an accuracy within 20 meters. With the network and fused location services, the $80 \%$ of data is within 40 meters and 38 meters respectively. The $83 \%$ of all the location points collected in our project has an accuracy within 40 meters.

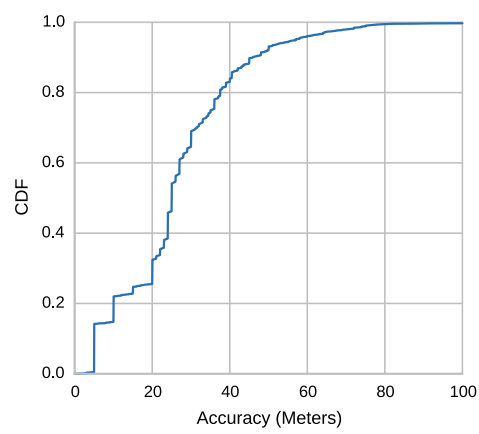

a)

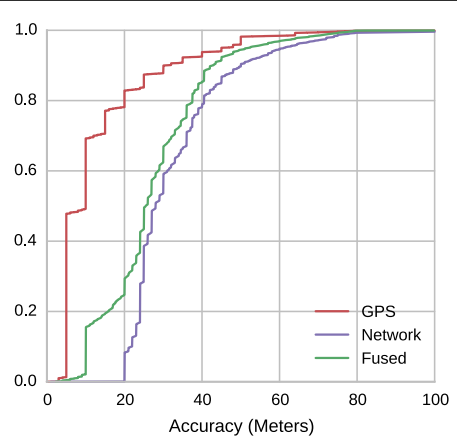

b)

Figure 2 Location points' accuracy. (a) Estimated cumulative distribution of the accuracy of all the collected location points; (b) Estimated cumulative distribution of the accuracy of all the location points collected, divided by their respective location provider: GPS, Network and Fused Location Provider. 


\subsubsection{Spending data}

A large body of research has shown that individuals with more money are happier [40, 41], although this relationship is weaker than many people expect [42-44]. Recent studies in social and positive psychology suggest that how people spend their money may be at least as important as the amount of money they have [45]. More specifically, investing discretionary resources into life experiences rather than buying material possessions, seems to make people happier [46-48]. Moreover, both correlational and experimental studies have shown that people who spend money on others report more happiness [49]. For this reason, determining an individual's purchasing style (e.g., whether the individual tends to spend money on life experiences or material items, or whether the individual tends to spend money on others) may be useful to infer life satisfaction and subjective happiness.

In our study, we collected spending behaviours by means of an experience sampling [50] smartphone application that allows users to report their daily expenses. Thus, from May 1, 2013 to July 31, 2013, 69 study participants were asked each day to indicate all the expenses they made. For each expense we collected the user information, the category of the expense (see Table 2), the type, the amount, the timestamp and the eventual notes they were willing to write and share. The type of the expenses are listed in Table 1.

\subsubsection{Personality and individual traits}

Upon joining the lab each participant filled out several questionnaires to collect information about her/his psychological traits and dispositions (e.g. Big Five personality traits, Subjective Happiness, Satisfaction with Life, etc.).

Scientific psychology has developed a view of personality as a stable high-level abstraction, introducing the notion of personality traits which are stable dispositions towards action, belief and attitude formation. The main assumption is that personality traits differ across individuals, are relatively stable over time and influence behaviour [51]. Interindividual differences in behaviour, belief and attitude can therefore be captured in terms of the dispositions/traits that are specific to each individual. This provides a powerful descriptive and predictive tool that has been widely exploited by various disciplines including social, clinical, educational and organizational psychology.

The search for personality traits has often been pursued by means of factor-analytic studies applied to lists of trait adjectives [52]. A well-known and very influential example of a multi-factorial approach is the Big Five model $[53,54]$. This model takes five main traits as constitutive of people's personality: (i) Extraversion (sociable, assertive, playful), (ii) Agreeableness (friendly, cooperative), (iii) Conscientiousness (self-disciplined, organized), (iv) Emotional Stability (calm, unemotional), and (v) Openness to Experience. Over the last 50 years the Big Five model has become standard in psychology. In our study, Big Five personality traits are measured by means of Italian version of the Big Five Marker Scales (BFMS) questionnaire [55].

Table 1 Description of the expense typologies made by the participants in MTL community

\begin{tabular}{llc}
\hline Type & Description & Number of expenses \\
\hline 0 & The expense was made for something I need & 1,114 \\
1 & The expense was made for something my children need & 499 \\
2 & The expense was made for a gift & 261 \\
3 & The expense was made for something the family need & 1,533 \\
\hline
\end{tabular}


Last decades have seen an increase in research on subjective well-being, which refers to how people experiences the quality of their lives [56]. Research has identified two broad aspects of subjective well-being: (i) an affective component, which is usually further divided into positive and negative affect [56] and (ii) a cognitive component, which is referred to as life satisfaction [57]. In our living lab study, we collected life satisfaction information using the Satisfaction with Life Scale (SWLS) [56]. Life satisfaction refers to a judgmental process, in which individuals assess the quality of their lives on the basis of their own set of criteria [58]. Specifically, the SWLS is composed by five items (e.g. 'In most ways my life is close to my ideal, 'The conditions of my life are excellent', etc.), allowing the respondents to reach a global judgment of life satisfaction. In order to collect also a global evaluation of 'subjective happiness' - that is a global subjective evaluation of whether one is an happy or unhappy person - we resorted to the 4-items Subjective Happiness Scale (SHS) [59].

\subsection{My data store}

The ubiquitous collection of personal data raises unprecedented privacy challenges. Users have to make decisions concerning the disclosure of their personal information on the basis of a difficult tradeoff between data protection and the advantages stemming from data sharing. More importantly, people are typically not involved in the life-cycle of their own personal data, which results in a lack of understanding of who uses their data and for what. Several researchers have proposed and investigated new user-centric models for personal data management, which enable individuals to have more control of their own data's life-cycle [60]. To this end, researchers and companies are developing repositories which implement medium-grained access control to different kinds of personally identifiable information, such as e.g. passwords, social security numbers and health info [61], and more recently location $[62,63]$ and personal data collected by means of smartphones [63]. During the MTL study, we designed, developed and tested My Data Store [64], a digital space, owned and controlled through a Web interface by users, acting as repository for the personal information collected during the study and offering every user the possibility to view, control and disclose her/his own data. Data were organized in sections by putting together data having a similar meaning (e.g., data about locations were organized in the same region, independently of whether they were collected through GPS or a WiFi contact). One section of My Data Store is designed to provide users with visualizations of their personal data. Two types of individual views are available for each kind of owned data: (i) detailed views (in tables or maps), where every available piece of raw data is represented in detail, and (ii) aggregated views with aggregations, at different levels, of the personal data (e.g. charts, pies, clusters of frequent locations, quantity of contacts, etc.). My Data Store also features a sharing area, a space for subjects to fix the desired disclosure level of their data, distinguished into: (i) do not share, (ii) share anonymously, and (iii) share not anonymously (i.e. labeling the data with some personal demographic information). More details about My Data Store can be found in [64].

\section{Results}

All the results presented in the following sections are based on data collected between February 2013 and December 2014. Thanks to the variety of data at our disposal, different aspects of human behaviour such as patterns of communication, location and mobility, spending patterns and personality traits were studied and discussed. 


\subsection{Communication patterns}

During the monitored period we collected 612,856 phone calls. From them, only 332,037 calls were taken into account, which represent the subset of incoming and outgoing calls with duration longer than zero seconds. The average duration of the calls is $154.29 \mathrm{sec}-$ onds, with a median duration of 73.0 seconds. In the same period of time 342,814 SMS were collected. The average ratio between incoming and outgoing calls for a participant is $r_{\mathrm{in}_{\text {calls }} / \text { out }_{\text {calls }}}=0.88$, while for SMS the average ratio is $r_{\mathrm{in}_{\text {sms }} / \text { out }_{\text {sms }}}=2.96$. This indicates that a person receives almost three times more SMS than what he send. In this study we consider both communication interactions inside and outside the MTL community. Figure 3 shows the distribution of all communication interactions.

Generally, people perform different activities during the day. Many of them are repeated on a daily basis such as eating at noon, jogging in the evening etc., and others are repeated on a weekly basis (for example watching your favourite soccer team). In Figure 4 it is possible to observe a strong daily seasonality which usually starts around 7 am, when people generally turn on their phones and probably commute to work and then slowly decreases in the evening, when people return home and go to sleep. Moreover, there is also a weekly seasonality due to work cycles, with different communication patterns between working days and weekends, where it is possible to notice a decrease in the mean number of both SMS and phone calls. Another interesting thing to observe is represented by the different daily seasonality of SMS and calls. SMS are more used in the morning and decrease from noon onwards, while phone calls are more used in the evening hours. This could be explained by the fact that people during working hours, since they are working, are forced to use SMS, which is a faster and concise communication mean with respect to phone calls. As expected, at the end of a work day, we can see a significant rise on the mean number

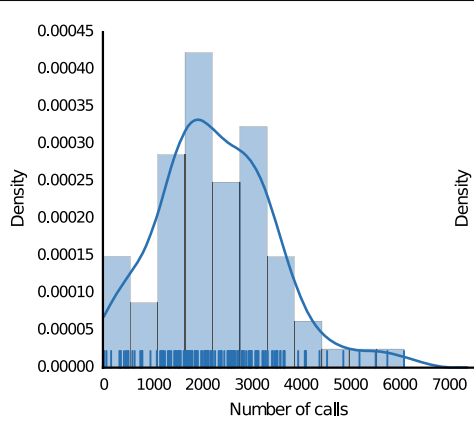

a)

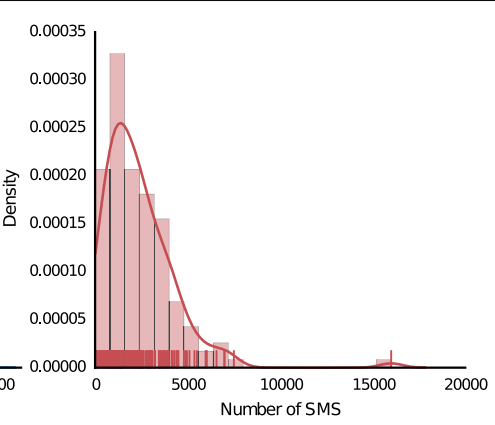

b)

Figure 3 Calls and SMS distributions. Distribution of the number of (a) calls and (b) SMS sent and received by participants of the MTL community. The distribution of calls is roughly similar to a Normal distribution, while the SMS one is heavily skewed with a peak around 1,500.

Figure 4 Weekly mobile phone activity behaviour. Number of calls and SMS exchanged in a typical weekday (both internal and external calls/SMS of the community are considered). The gray area represent the period of the day between 12 am and $12 \mathrm{pm}$.

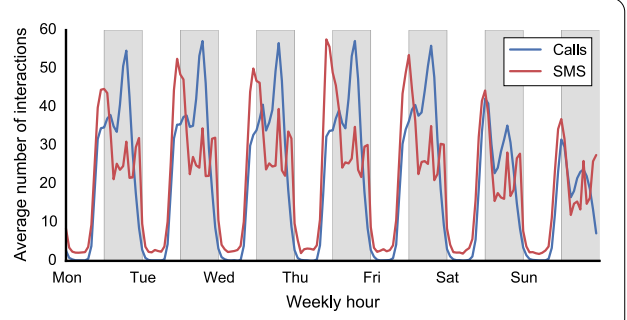


of phone calls. These patterns cannot be seen during the weekend, where the patterns of SMS and calls are very similar.

The number of unique SMS contacts per participant $\left(N_{S M S}\right)$ and the number of unique call contacts per participant $\left(N_{\text {call }}\right)$ have a Pearson correlation of 0.478 ( $p$-value $1.10 \times$ $\left.10^{-9}\right)$. Figure 5 shows this correlation. From the scatter plot it is possible to notice the presence of few outliers: we have some participants that did not use SMS at all. For example it is easy to see that there are two people who have done a lot of phone calls without sending SMS. If we consider only the participants with at least five SMS and calls in the whole period, the Pearson correlation between $N_{\mathrm{SMS}}$ and $N_{\text {calls }}$ rises to 0.661 ( $p$-value $9.89 \times$ $10^{-19}$ ). This means that excluding outliers, namely people who did not send any SMS or did not make any call, we have a very similar use of the phone during the considered period.

Moreover, we found that the Jaccard similarity coefficient between people which participants contacted via calls $\left(P_{\text {call }}\right)$ and via SMS $\left(P_{\text {SMS }}\right)$ is:

$$
J\left(P_{\text {call }}, P_{\mathrm{SMS}}\right)=\frac{\left|P_{\text {call }} \cap P_{\text {SMS }}\right|}{\left|P_{\text {call }} \cup P_{\text {SMS }}\right|}=0.276
$$

This degree of similarity suggests that participants in our community, even though they use both kind of communications means, they use SMS and phone calls for different purposes and in different ways.

Figure 6 shows an example of the interactions patterns on a single day (Monday, 18 March 2013) between the SMS and the voice calls communication channels. The networks are drawn with a spring-force layout, which groups the participants which are more interconnected among each others. The participants that are not strongly interconnected tend to be drawn further apart. We decided to evaluate the activations network in four time slots. Please note that the activation links during the night (00:00-06:59) are very sparse, while during the rest of the day the interactions become more and more interconnected, i.e. the graph is denser with a larger number of edges.

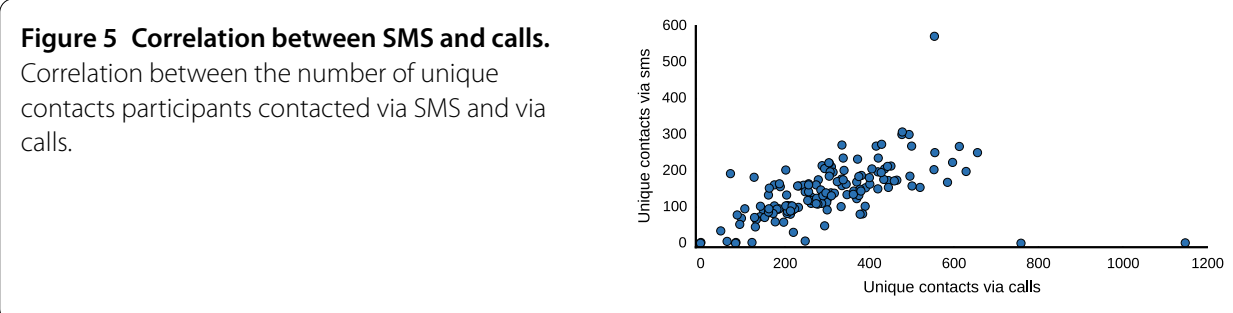

Figure 6 Mobile phone daily activations. Daily activation network in calls (blue) and SMS (red) interactions considering both internal and external participants. It shows the activation links on a Monday (Monday, 18 March 2013) in four different time slots of 6 hours.

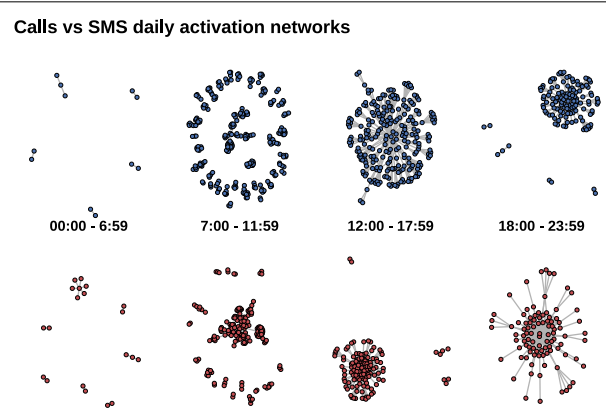



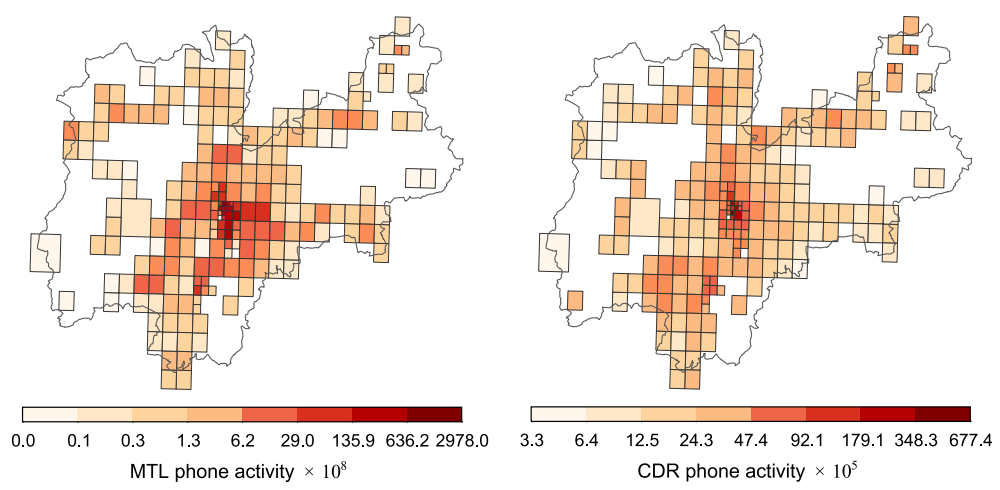

Figure 7 Choroplet maps of mobile activity density. (a) Choropleth map of the MTL activity density measured with the number of calls; (b) Choropleth map of the CDRs phone activity density measured with the number of calls.

Mobile phone activity can be also observed in its spatial dimension. Hence, in Figure 7 we compare the spatial behaviour of the MTL community with the entire population's behaviour extracted from the Call Detail Records (CDRs) of the Trentino province during the same period of the MTL living lab project. CDRs are collected by mobile phone companies for billing purposes. Every time a mobile phone engages in a telecommunication interaction, a Radio Base Station (RBS) is assigned by the operator in order to deliver the communication through the network. Then, a new CDR is created recording the time of the interaction and the RBS which handled it. Despite the first intuitive similarity showed on the map, the MTL community is very active near the biggest cities (mainly Trento), placed in the center of the map.

\subsection{Location and mobility}

Human trajectories are far from being random, showing a high degree of temporal and spatial regularity. This is mainly due to the fact that people spend most of their time in a small set of locations. For this reason, for all participants in our community we computed the radius of gyration [16] defined as:

$$
r_{g}=\sqrt{\frac{1}{n} \sum_{i=1}^{n}\left(r_{i}-r_{\mathrm{cm}}\right)^{2}},
$$

where $n$ is the number of points, $r_{i}$ is the position recorded as longitude and latitude and $r_{\mathrm{cm}}$ is the center of mass of the trajectories, defined as:

$$
r_{\mathrm{cm}}=\frac{1}{n} \sum_{i=1}^{n} r_{i}
$$

Figure 8(a) shows that the radius of gyration's distribution is represented by a truncated power law distribution, with a peak around $100 \mathrm{~km}$. This means that the majority of users in our project moved in a range of $100 \mathrm{~km}$ during the considered period.

Figure 9 shows the mobility patterns of the participants in our community inside the Trentino area, using a 2-D histogram of the location points with hexagonal cells. Each 


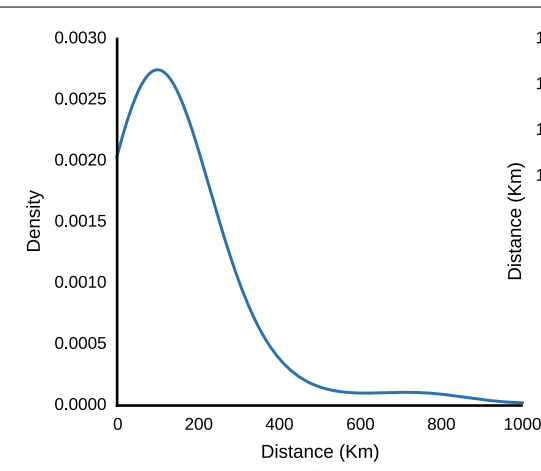

a)

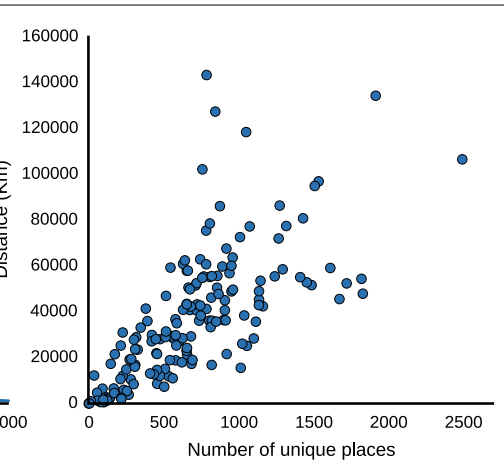

b)

Figure 8 Radius of gyration and unique places' correlation with distance. (a) Kernel Density Estimation of users' radius of gyration. As it can be seen, the majority of the users have a radius of gyration around 100 km; (b) Total number of unique places, computed clustering together stay points within a range of 100 meters, and total distance travelled by each MTL participant.

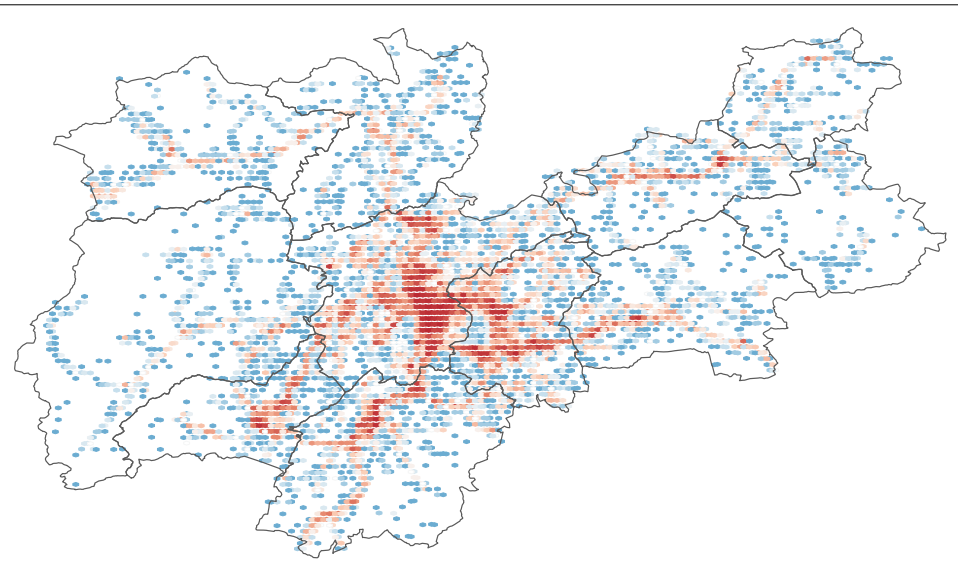

Figure 9 Mobility patterns in Trentino. Mobility patterns in Trentino with logarithmic color scale. Each hexagonal bin is coloured (from blue to red) accordingly to the number of location points that fall within that hexagon.

hexagon is coloured (from blue to red) accordingly to the number of location points that fall within that hexagon. As expected, the majority of location points were collected in the city of Trento, which being the largest city of Trentino offers more services, cultural, social life and work opportunities. The other most frequented places are distributed along the Trentino valleys where the majority of people live.

From the collected location points, it is possible to estimate stay points. A stay point is defined as a geographic region in which a user stays for a certain amount of time [65]. Therefore, each stay point carries its semantic meaning, that is for instance, the living and working places, the restaurant and shopping mall a person visited etc. These places are found iteratively seeking the spatial area in which an individual stays for a period over a threshold (see Figure 10).

In our study we detected stay points with the algorithm explained in [65], defining a stay point as a place where a participant remained within a range of 100 meters for at least 15 minutes. 


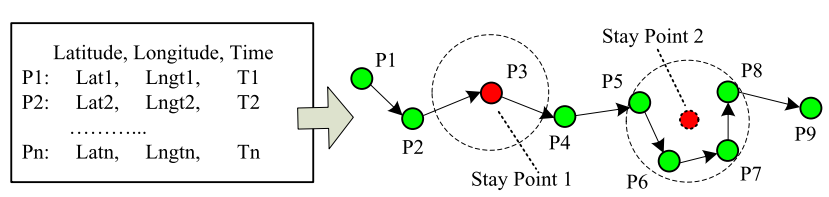

Figure 10 Stay points. Stay points extracted from locations points. We defined a stay point as the place where a participant remained within a range of 100 meters for at least 15 minutes. Figure from Ye et al. [65].

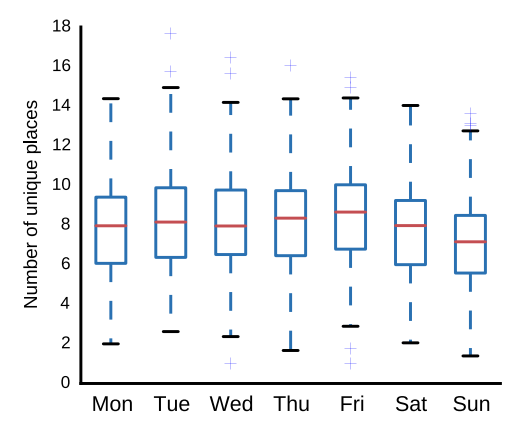

a)

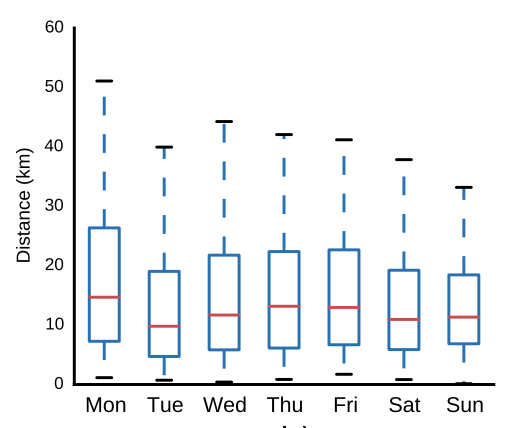

b)

Figure 11 Weekly mobility behaviour. Boxplots of the number of unique places visited (a) and the distance travelled by the MTL participants (b) in a typical weekday.

We also studied weekly temporal dynamics of the distance and the number of unique places of a generic participant. As shown in Figure 11 the median number of unique places visited by a participant is stable over the week with the exception of the weekend when people visit fewer places. This is probably due to the fact that people over the weekend do not commute to their workplace, thus potentially visiting less places. Moreover, the day when people tend to travel more is Monday. Despite these little variations, either the number of unique places and the travelled distance seem very stable within a week.

We also investigated the total distance travelled and the number or total unique stay points visited by each participant in the observation period (two years). In order to estimate the unique stay points visited by each user we clustered all the stay points using the density based clustering algorithm DBSCAN [66]. This algorithm groups points that are closely packed together with two parameters: $\epsilon$ which defines the maximal size of the cluster, and minPts namely the minimum number of points required to form a dense region. In our experiment we used $\epsilon=100$ meters as the stay points' range threshold and minPts $=1$ in order to consider also places with just one stay point. Figure 8(b) shows the relation between unique places and total travelled distance which are very related among each other with a Spearman's rank of 0.70 with $p$-value $7.3 \times 10^{-26}$. This highlights the stability between the two dimensions.

\subsection{Spending patterns}

We collected 3,407 expenses from 69 participants. On average a user spent 60.55 euro, with a standard deviation of 474.38 euro. The cheaper collected expense was about food for 0.22 euro and the most expensive one (22,000 euro) is probably for a car. Table 2 shows the detailed spending statistics. 
Figure 12 shows that the expenses' distribution is skewed. Thus, people tend to have a large number of small expenses relative to the total amount of money spent. Similarly, in the same figure it is possible to observe the relation of the number of expenses and the median amount of money spent by each user. The large majority of people usually has small purchases, while some users are characterized by few big expenses.

Figure 13 represents the total amount of spending for each category. Note that the highest expenses correspond to the transportation category (related to motorbikes or cars), followed in order by food, holiday and home categories. Since the transportation category is biased by the high cost of the three expenses aforementioned, we can easily say that in our community the highest number of spending events and amounts correspond to basic

Table 2 Statistics of spending events done by 69 participants of our community per each spending category

\begin{tabular}{|c|c|c|c|c|c|c|}
\hline Category & Count & Amount & Mean & Std. dev. & Min & Max \\
\hline Food & 1,386 & $36,333.57$ & 26.21 & 39.42 & 0.22 & 1,000 \\
\hline Health & 178 & $6,360.78$ & 35.73 & 38.12 & 5.00 & 277 \\
\hline Hobbies & 297 & $9,257.02$ & 31.17 & 53.49 & 1.00 & 480 \\
\hline Home & 229 & $27,442.76$ & 119.84 & 296.08 & 0.80 & 3,700 \\
\hline Transportation & 400 & $64,886.37$ & 162.22 & $1,335.43$ & 0.50 & 22,000 \\
\hline Technology & 49 & $6,177.63$ & 126.07 & 210.92 & 0.89 & 1,100 \\
\hline Holiday & 148 & $30,478.75$ & 205.94 & 344.27 & 1.30 & 1,800 \\
\hline Wellbeing & 154 & $5,876.61$ & 38.16 & 39.75 & 1.50 & 235 \\
\hline Clothes & 342 & $14,913.74$ & 43.61 & 52.20 & 0.88 & 500 \\
\hline Social & 224 & $4,564.43$ & 20.38 & 24.01 & 0.35 & 150 \\
\hline
\end{tabular}

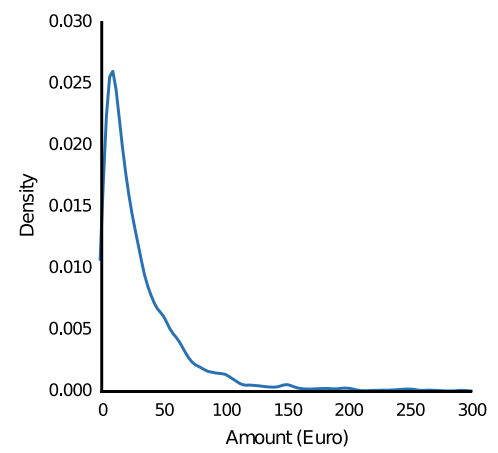

a)

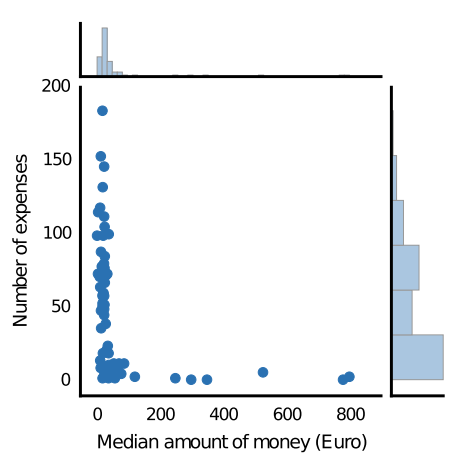

b)

Figure 12 Participants' spending behaviour. (a) Distribution of the amount of money spent in each single spending event. For visual reason, the distribution is truncated at 300 euro; (b) Relation between the number of expenses per user and the median amount of money spent. It is possible to observe that users who spent a lot are those who did not enter a lot of expenses in the application.

Figure 13 Spending categories. Total amount of money spent in each category. Although the number of spending events in the transportation category is small, this category is biased by the high cost of three expenses inserted (probably due to the purchase of cars and/or motorbikes).

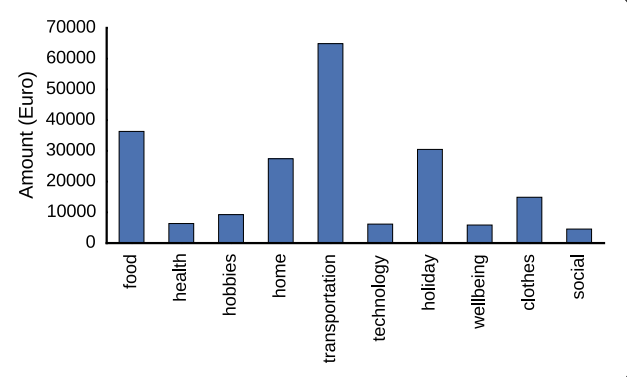


needs, such as food. This is in line with the recent survey on families done by the Italian national institute of statistics [67] (access date 09/11/2015), which observed that food and beverages expenses are the highest, if we exclude transportation and households expenses, further supporting the idea that MTL-like methods for collecting actual behavior can complement and even substitute traditional survey-based ones.

All the other basic statistics on each category are represented in Table 2.

\subsection{Personality traits and other individual dispositions}

140 participants actively filled the personality traits specified in the questionnaire provided in the survey. Two of them did not complete the survey so we excluded them from this part of the analysis. Since personality affects people mobile phone usage [68], communication $[20,21,69,70]$ and mobility patterns [21] and people purchasing styles [71], we investigated the possible relationships between personality traits and communications, mobility and the spending patterns of the MTL study participants. The distribution of each personality trait is shown in Figure 14 .

\subsubsection{Communications}

Table 3 reports the Spearman's rank correlation between mobile phone interactions (calls and SMS) and personality traits. On average, extroverted individuals call more often than introverts, and extroverts also show both daily and weekly stability. In fact, they enjoy interacting with people, and are often perceived as being full of energy. Hence, other people seem to be entertained by conversing with them. On the other hand, introverted individuals seem to be less regular in their patterns of calling.

The results for Conscientiousness indicate that people with a preference for planned rather than spontaneous behavior tend to receive fewer SMS messages from others. Emotionally unstable people are in contact with more individuals and tend to send and receive more SMS messages. Moreover, they are more likely to have longer phone conversations. As expected, emotionally unstable people are less regular in their SMS communications. This personality trait is, overall, the most significant factor related to communication interactions.

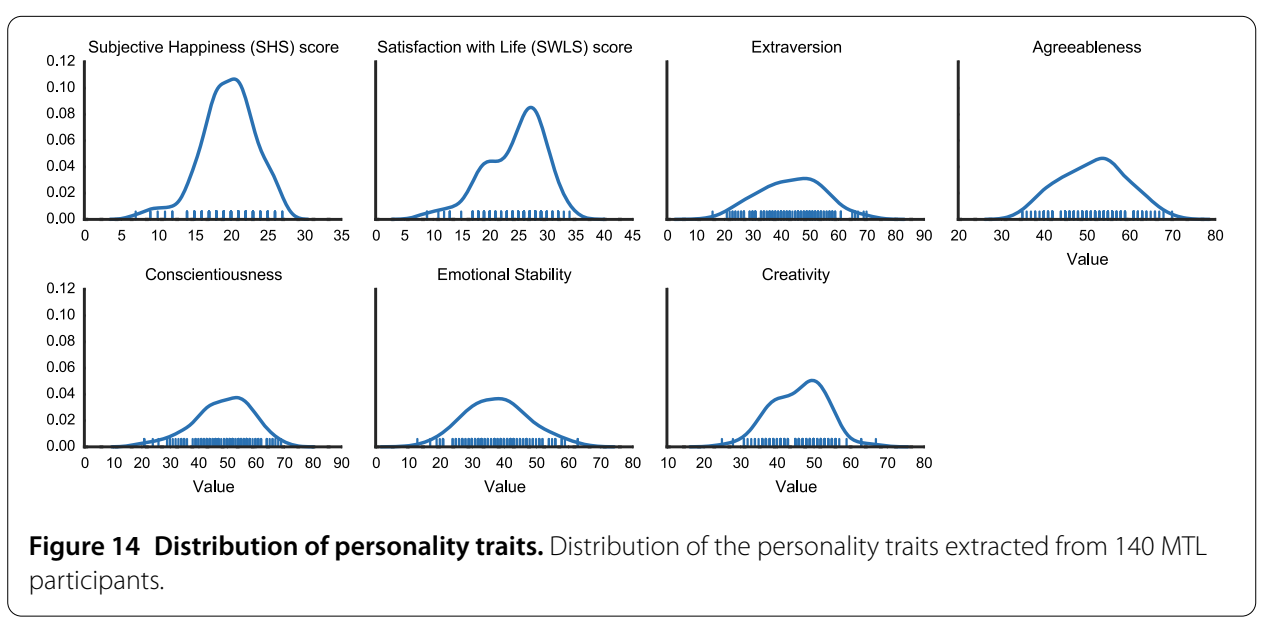


Table 3 Spearman's rank correlation between the mobile interactions and the personality attributes

\begin{tabular}{|c|c|c|c|c|c|}
\hline & Extraversion & Agreeableness & Conscientiousness & $\begin{array}{l}\text { Emotional } \\
\text { stability }\end{array}$ & $\begin{array}{l}\text { Openness to } \\
\text { experience }\end{array}$ \\
\hline \multicolumn{6}{|l|}{ Incoming calls } \\
\hline avg. unique people (daily) & & & & & $-0.169^{*}$ \\
\hline avg. unique people (weekly) & & & & & $-0.185^{*}$ \\
\hline avg. num. calls (total) & & & & $-0.280^{* * *}$ & \\
\hline total duration & & & & $-0.318^{* * *}$ & \\
\hline total unique people & & & & & $-0.172^{*}$ \\
\hline \multicolumn{6}{|l|}{ Outgoing calls } \\
\hline avg. num. calls (daily) & $0.169^{*}$ & & & & \\
\hline std. num. calls (daily) & $0.199^{*}$ & & & & \\
\hline avg. unique people (daily) & $0.192^{*}$ & & & & \\
\hline std. unique people (daily) & $0.278^{* * *}$ & $0.174^{*}$ & & & \\
\hline avg. num. calls (weekly) & $0.169^{*}$ & & & & \\
\hline std. num. calls (weekly) & $0.174^{*}$ & & & & \\
\hline avg. unique people (weekly) & $0.221^{* *}$ & $0.190^{*}$ & & & \\
\hline std. unique people (weekly) & $0.240^{* *}$ & & & & \\
\hline total num. calls & & & & $-0.206^{*}$ & \\
\hline total duration & & & & $-0.259^{* *}$ & \\
\hline \multicolumn{6}{|l|}{ Incoming SMS } \\
\hline avg. num. SMS (daily) & & & $-0.234^{* *}$ & $-0.386^{* * *}$ & \\
\hline std. num. SMS (daily) & & & $-0.238^{* *}$ & $-0.362^{* * *}$ & \\
\hline avg. unique people (daily) & & & $-0.211^{*}$ & $-0.359^{* * *}$ & \\
\hline std. unique people (daily) & & & $-0.182 *$ & $-0.272^{* *}$ & \\
\hline avg. num. SMS (weekly) & & & $-0.234^{* *}$ & $-0.387^{* * *}$ & \\
\hline std. num. SMS (weekly) & & & $-0.276^{* * *}$ & $-0.364^{* * *}$ & \\
\hline avg. unique people (weekly) & & & & $-0.321^{* * *}$ & \\
\hline total num. SMS & $-0.168^{*}$ & & $-0.239 * *$ & $-0.458^{* * *}$ & \\
\hline total unique people & & & $-0.178^{*}$ & $-0.285^{* * *}$ & \\
\hline \multicolumn{6}{|l|}{ Outgoing SMS } \\
\hline avg. num. SMS (daily) & & & & $-0.232^{* *}$ & \\
\hline std. num. SMS (daily) & & & & $-0.212^{*}$ & \\
\hline avg. unique people (daily) & & & & $-0.199 *$ & \\
\hline std. unique people (daily) & & & & $-0.178^{*}$ & \\
\hline avg. num. SMS (weekly) & & & & $-0.235^{* *}$ & \\
\hline std. num. SMS (weekly) & & & & $-0.211^{*}$ & \\
\hline avg. unique people (weekly) & & & & $-0.213^{*}$ & \\
\hline total num. SMS & & & & $-0.310^{* * *}$ & \\
\hline total unique people & & & & $-0.225^{* *}$ & \\
\hline
\end{tabular}

Blank cells indicate not significant correlations. Note: ${ }^{*} p<0.05, * * p<0.01, * * * p<0.001$.

\subsubsection{Mobility}

We also investigated the relationship between mobility patterns and personality traits. We found a positive correlation $(0.171 p$-value $<0.05)$ between the average number of daily unique places per participant and the Emotional Stability trait. People with higher Emotional Stability tend to have a higher average number of daily visited places when compared with people with lower Emotional Stability scores. This suggests that in our study the more calm and emotionally stable a person is, the higher the number of daily visited places.

Surprisingly, the total number of places per participant is negatively correlated with the Extraversion personality trait $(-0.207 p$-value $<0.05)$ and the Emotional Stability trait $(-0.188 p$-value $<0.05)$. Extroverts tend to visit few places with respect to more introverted people. The same is true for people with higher Emotional Stability; in fact they 
present a lower number of total visited places with respect to their counterpart. These results require further investigation.

In the same way we explored if there are associations between the personality traits and the radius of gyration. We found a negative correlation between the daily radius of gyration per participant and the Extraversion personality trait $(-0.175 p$-value $<0.05)$ and the Emotional Stability trait $(-0.250 p$-value $<0.01)$. Extroverts and people with higher Emotional Stability tend to have a daily radius of gyration smaller than their counterpart.

Finally, we did not find any significant association between personality traits and the total and daily travelled distance.

\subsubsection{Spending}

In this section, we report only the significant correlations we found between personality traits and other individual dispositions (e.g. life satisfaction and subjective happiness) and the purchasing styles of the study participants. For example, the Subjective Happiness Score (SHS) is negatively correlated $(-0.329 p$-value $<0.05)$ with the number of spending events related the category 'gift for the kids'. This means that people with a lower SHS, namely reporting to feel less happy, tend to buy more gifts with respect to people with a higher SHS, namely happy people. At the same time, people with a large average amount of money spent to purchase something for their children, tend to be more satisfied with their life (correlation $0.305 p$-value < 0.05). Similarly, happy people seems to spend on average more for their children than people less satisfied about their life. No correlation was found between the Big Five personality traits and the spending patterns of the study participants.

\section{Discussion}

In this paper, we have described the Mobile Territorial Lab (MTL) project, a longitudinal living lab study that sensed the lives of more than 100 parents distributed in different areas of Trentino, and the results after two years of experimentation. Through the use of mobile phones as an active sensing platform, this project provides, to the best of our knowledge, the most complete picture of the daily lives of parents of young children.

Previous studies were usually focused on students' lives and on places such as dorms and university campuses. Here, we collected high resolution data that consisted of mobile phone interactions (calls and SMSs), mobility, spending, and individual dispositions and personality traits data, that allowed us to derive multi-view insights on parents' daily lives.

From our analyses, we were able to observe the weekly seasonality and the different communication patterns between working days and weekends. Parents' movements were fairly predictable in their aggregated view. In fact, despite we collected data about people with different incomes and working habits, their movements are concentrated around a radius of about 100 kilometers showing also a regularity on the number of places visited during the week. This is probably explained by the cyclic rhythm of families with children, which is composed by work-time activities and a mix of leisure and family duties that forces the regularity of the movements' patterns. Moreover, as shown by our analyses, they tend to move from/to the Trento area, which is the area that offers more services, cultural, social life and work opportunities with respect to the surrounding valleys. We also observed the substantial correlations that travel, spending and social relations are modulated by 
personality. Especially Extraversion and Emotional Stability seem to influence all the observed behaviours. While we provide some supporting evidence of this connection, there is still need for further investigation.

Overall, our results prove the relevance of these living lab approaches to measure human behaviors and interactions, which can pave the way to new studies exploiting a richer number of behavioral indicators. An example is the ongoing Kavli HUMAN project [72] that is planning to gather socio-demographic information, medical records, genetic and microbiome data, patterns of physical activity and communication interactions from mobile sensing, personality traits and other individual dispositions, etc. for 10,000 people living in New York City.

Our approach can also overcome some of the limitations of traditional research methods in social science based on surveys or ethnographic observation [73]. Hence, the newly arised field of Computational Social Science, of which our study is a notable example, represents a great opportunity to identify insights and methods that can be used next to classical social science approaches. Not only this improves the granularity and the effectiveness of behavioural studies designed only with classical approaches, but also it overcomes the expensive data collection in the long term.

Finally, we believe that the collected data could be very relevant for researchers from different disciplines such as social psychology, sociology, computer science, economy, anthropology, etc., which are interested in understanding human behaviour. For example, computer scientists can design machine learning algorithms to predict purchasing styles and spending behaviors from communication and mobility patterns $[19,74$, 75]. Economists and social psychologists can investigate the relationship between actual spending behavior and life satisfaction and happiness [45]. Social psychologists, jointly with computer scientists, can design approaches to predict stress and depression from communication and mobility patterns before they could cause severe problems [24, 25, 27]. For this reason, we are actively working on making this data available to everyone through an Open Database License (ODbL), as we previously did for the aggregated Call Detail Records (CDRs) of the Trento province [76].

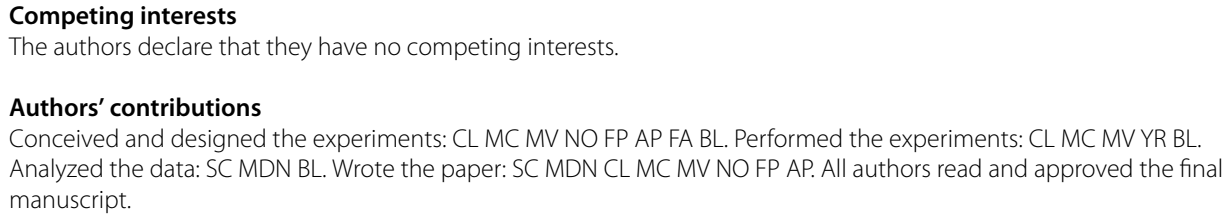

\section{Acknowledgements}

We thank all the study participants. The implementation of the mobile sensing platform and the data collection process are the results of the work of the following authors: Mattia Pasolli, Silvana Bernaola, Andrea Cappellotto, Lorenzo Waldner, and Jeff Schmitz. The community management process is the result of the work of Nicola Doppio, Erica Paris, and Daniela Stefanescu. Finally, the design of the study is the result also of the contributions of Corrado Moiso, Massimo Zancanaro and John Henry Clippinger.

Received: 15 November 2015 Accepted: 8 February 2016 Published online: 20 February 2016

References

1. Goffman E (1959) The presentation of self in everyday life. Doubleday, New York 
2. de Certeau M (1984) The practice of everyday life. University of California Press, Berkeley

3. Schensul SL, Schensul JJ, LeCompte MD (1999) Essential ethnographic methods: observations, interviews, and questionnaires, vol 2. Rowman Altamira, Lanham

4. Brown E, Shortell T (2014) Walking in the European city: quotidian mobility and urban ethnography. Ashgate Publishing, Ltd, Farnham

5. Hymes D (1964) Introduction: toward ethnographies of communication. Am Anthropol 66(6):1-34

6. Geertz C (1973) The interpretation of cultures: selected essays. Basic Books, New York

7. O'Reilly K (2012) Ethnographic methods. Routledge, Abingdon

8. Lane ND, Miluzzo E, Lu H, Peebles D, Choudhury T, Campbell AT (2010) A survey of mobile phone sensing. IEEE Commun Mag 48(9):140-150

9. Miller G (2012) The smartphone psychology manifesto. Perspectives Psychol Sci 7(3):221-237

10. Dong W, Lepri B, Pentland A (2011) Modeling the co-evolution of behaviors and social relationships using mobile phone data. In: MUM, pp 134-143

11. Aharony N, Pan W, Ip C, Khayal I, Pentland A (2011) Social fMRI: investigating and shaping social mechanisms in the real world. Pervasive Mob Comput 7(6):643-659

12. Madan A, Cebrian M, Moturu S, Farrahi K, Pentland A (2012) Sensing the "health state of a community". IEEE Pervasive Comput 4:36-45

13. Stopczynski A, Sekara V, Sapiezynski P, Cuttone A, Madsen MM, Larsen JE, Lehmann S (2014) Measuring large-scale social networks with high resolution. PLoS ONE 9(4):e95978

14. Lazer D, Pentland A, Adamic L, Aral S, Barabasi A-L, Brewer D, Christakis N, Contractor N, Fowler J, Gutmann M, Jebara T, King G, Macy M, Roy D, Van Alstyne M (2009) Computational social science. Science 323(5915):721-723

15. King G (2011) Ensuring the data-rich future of the social sciences. Science 331(6018):719-721

16. Gonzalez MC, Hidalgo CA, Barabasi A-L (2008) Understanding individual human mobility patterns. Nature 453(7196):779-782

17. De Domenico M, Lima A, Musolesi M (2013) Interdependence and predictability of human mobility and social interactions. Pervasive Mob Comput 9(6):798-807

18. Do TMT, Dousse O, Miettinen M, Gatica-Perez D (2015) A probabilistic kernel method for human mobility prediction with smartphones. Pervasive Mob Comput 20:13-28

19. Singh VK, Freeman L, Lepri B, Pentland A (2013) Predicting spending behavior using socio-mobile features. In: 2013 international conference on social computing (SocialCom), pp 174-179

20. Staiano J, Lepri B, Aharony N, Pianesi F, Sebe N, Pentland A (2012) Friends don't lie: inferring personality traits from social network structure. In: Proceedings of the 2012 ACM international joint conference on pervasive and ubiquitous computing (UbiComp'12). ACM, New York, pp 321-330

21. de Montjoye Y-A, Quoidbach J, Robic F, Pentland A (2013) Predicting personality using novel mobile phone-based metrics. In: Social computing, behavioral-cultural modeling and prediction, pp 48-55

22. Pielot M, Dingler T, Pedro JS, Oliver N (2015) When attention is not scarce-detecting boredom from mobile phone usage. In: Proceedings of the 2015 ACM international joint conference on pervasive and ubiquitous computing (UbiComp'15). ACM, New York, pp 825-836

23. Madan A, Cebrian M, Lazer D, Pentland A (2010) Social sensing for epidemiological behavior change. In: Proceedings of the 2010 ACM international joint conference on pervasive and ubiquitous computing (UbiComp'10). ACM, New York, pp 291-300

24. LiKamWa R, Liu Y, Lane ND, Zhong L (2013) MoodScope: building a mood sensor from smartphone usage patterns. In: Proceedings of the 11 th annual international conference on mobile systems, applications, and services (MobiSys'13), pp 389-402

25. Bogomolov A, Lepri B, Ferron M, Pianesi F, Pentland A (2014) Daily stress recognition from mobile phone data, weather conditions and individual traits. In: Proceedings of the ACM international conference on multimedia. ACM, New York, pp 477-486

26. Wang R, Chen F, Chen Z, Li T, Harari G, Tignor S, Zhou X, Ben-Zeev D, Campbel AT (2014) StudentLife: assessing mental health, academic performance and behavioral trends of college students using smartphones. In: Proceedings of the 2014 ACM international joint conference on pervasive and ubiquitous computing (UbiComp'14). ACM, New York, pp 3-14

27. Canzian L, Musolesi M (2015) Trajectories of depression: unobtrusive monitoring of depressive states by means of smartphone mobility traces analysis. In: Proceedings of the 2015 ACM international joint conference on pervasive and ubiquitous computing (UbiComp'15). ACM, New York, pp 1293-1304

28. Eagle N, Pentland A (2006) Reality mining: sensing complex social systems. Pers Ubiquitous Comput 10(4):255-268

29. Savin-Williams RC, Small SA (1986) The timing of puberty and its relationship to adolescent and parent perceptions o family interactions. Dev Psychol 22(3):342-347

30. Nelson SK, Kushlev K, English T, Dunn E, Lyubomirsky S (2013) In defense of parenthood: children are associated with more joy than misery. Psychol Sci 24:3-10

31. Evenson RJ, Simon RW (2005) Clarifying the relationship between parenthood and depression. J Health Soc Behav 46:341-358

32. Devault M (2000) Producing family time: practices of leisure activity beyond the home. Qual Sociol 23(4):485-503

33. Umberson D, Gove WR (1989) Parenthood and psychological well-being: theory, measurement, and stage in the family life course. J Fam Issues 10:440-462

34. Nelson SK, Kushlev K, Lyubomirsky S (2014) The pains and pleasures of parenting: when, why, and how is parenthood associated with more or less well-being? Psychol Bull 140(3):846-895

35. Staiano J, Oliver N, Lepri B, de Oliveira R, Caraviello M, Sebe N (2014) Money walks: a human-centric study on the economics of personal mobile data. In: Proceedings of the 2014 ACM international joint conference on pervasive and ubiquitous computing (UbiComp'14). ACM, New York, pp 583-594

36. Massa P, Leonardi C, Lepri B, Pianesi F, Zancanaro M (2015) If you are happy and you know it, say "I'm here": investigating parents' location-sharing preferences. In: Proceedings of INTERACT, pp 315-332

37. Goodman L (1961) Snowball sampling. Ann Math Stat 32:148-170 
38. Smart Crowds, Trento Territorial Lab. http://www.smartcrowds.net

39. Blondel V, Decuyper A, Krings G (2015) A survey of results on mobile phone datasets analysis. EPJ Data Sci 4(1):10

40. Diener E, Ng W, Harter J, Arora R (2010) Wealth and happiness across the world: material prosperity predicts life evaluation, whereas psychosocial prosperity predicts positive feeling. J Pers Soc Psychol 99(1):52-61

41. Diener E, Tay L, Oishi S (2013) Rising income and the subjective well-being of nations. J Pers Soc Psychol 104(2):267-276

42. Easterlin RA (1974) Does economic growth improve the human lot? Some empirical evidence. In: David PA, Reder MA (eds) Nations and households in economic growth: essays in honor of Moses Abramovitz. Academic Press, New York

43. Aknin LB, Norton MI, Dunn EW (2009) From wealth to well-being? Money matters, but less than people think. J Positive Psychol 4(6):523-527

44. Kahneman D, Krueger AB, Schkade D, Schwarz N, Stone AA (2006) Would you be happier if you were richer? A focusing illusion. Science 312(5782):1908-1910

45. Dunn E, Norton M (2013) Happy money: the science of smarter spending. Simon \& Schuster, New York

46. Carter TJ, Gilovich T (2010) The relative relativity of material and experiential purchases. J Pers Soc Psychol 98(1):146-159

47. Carter TJ, Gilovich T (2012) I am what I do, not what I have: the differential centrality of experiential and material purchases to the self. J Pers Soc Psychol 102(6):1304-1317

48. Howell RT, Hill G (2009) The mediators of experiential purchases: determining the impact of pyschological needs satisfaction and social comparison. J Positive Psychol 4:511-522

49. Dunn E, Aknin L, Norton M (2014) Prosocial spending and happiness: using money to benefit others pays off. Curr Dir Psychol Sci 23(1):41-47

50. Larson R, Csikszentmihalyi M (1983) The experience sampling method. In: New directions for methodology of social and behavioral science, vol 15. Jossey-Bass, San Francisco, pp 41-56

51. Matthews G, Campbell SE (2009) Sustained performance under overload: personality and individual differences in stress and coping. Theor Issues Ergon 10(5):417-442

52. Allport GW, Odbert HS (1936) Trait-names: a psycho-lexical study. In: Psychological monographs, vol 47. Psychological Review Company, Albany

53. Costa PT, McCrae RR (1992) Four ways five factors are basic. Pers Individ Differ 13(6):653-665

54. John OP, Srivastava S (1999) The Big Five trait taxonomy: history, measurement, and theoretical perspectives. In: Handbook of personality: theory and research, vol 2. Guilford Press, New York, pp 102-138

55. Perugini M, Di Blas L (2002) The Big Five Marker Scales (BFMS) and the Italian AB5C taxonomy: analyses from an emic-etic perspective. In: De Raad B, Perugini M (eds) Big Five assessment. Hogrefe \& Huber, Göttingen

56. Diener ED, Emmons RA, Larsen RJ, Griffin S (1985) The satisfaction with life scale. J Pers Assess 49(1):71-75

57. Andrews FM, Withey SB (1976) Social indicators of well-being: America's perception of life quality. Plenum Press, New York

58. Shin DC, Johnson DM (1978) Avowed happiness as an overall assessment of quality of life. Soc Indic Res 5:475-492

59. Lyubomirsky S, Lepper HS (1999) A measure of subjective happiness: preliminary reliability and construct validation. Soc Indic Res 46(2):137-155

60. Pentland A (2012) Society's nervous system: building effective government, energy, and public health systems. IEEE Comput 45(1):31-38

61. Want R, Pering T, Danneels G, Kumar M, Sundar M, Light J (2002) The personal server: changing the way we think about ubiquitous computing. In: Proceedings of the 2002 ACM international joint conference on pervasive and ubiquitous computing (UbiComp'02). Springer, London, pp 194-209

62. Hong JJ, Landay JA (2004) An architecture for privacy-sensitive ubiquitous computing. In: Proceedings of the 2 nd international conference on mobile systems, applications, and services (MobiSys'04). ACM, New York, pp 177-189

63. de Montjoye Y-A, Shmueli E, Wang SS, Pentland A (2014) OpenPDS: protecting the privacy of metadata through safeAnswers. PLoS ONE 9(7):e98790

64. Vescovi M, Lepri B, Perentis C, Moiso C, Leonardi C (2014) My data store: toward user awareness and control on personal data. In: Proceedings of the 2013 ACM international joint conference on pervasive and ubiquitous computing (UbiComp'13). ACM, New York, pp 179-182

65. Ye Y, Zheng Y, Chen Y, Feng J, Xie X (2009) Mining individual life pattern based on location history. In: Proceedings of the 2009 tenth international conference on mobile data management: systems, services and middleware (MDM'09). IEEE Comput Soc, Washington, pp 1-10

66. Ester M, Kriegel H-P, Sander J, Xu X (1996) A density-based algorithm for discovering clusters in large spatial databases with noise. In: Proceedings of the second international conference on knowledge discovery and data mining (KDD'96). AAAI Press, Menlo Park, pp 226-231

67. ISTAT, I consumi delle famiglie. http://www.istat.it/it/archivio/127996

68. Butt S, Phillips JG (2008) Personality and self reported mobile phone use. Comput Hum Behav 24:346-360

69. Chittaranjan G, Blom J, Gatica-Perez D (2011) Who's who with big-five: analyzing and classifying personality traits with smartphones. In: Proceedings of the 2011 15th annual international symposium on wearable computers (ISWC'11). IEEE Comput Soc, Washington, pp 29-36

70. Chittaranjan G, Blom J, Gatica-Perez D (2013) Mining large-scale smartphone data for personality studies. Pers Ubiquitous Comput 17(3):433-450

71. Howell RT, Pchellin P, lyer R (2012) The preference for experiences over possessions: measurement and construct validation of the experiential buying tendency scale. J Positive Psychol 7(1):57-71

72. Azmak O, Hannah B, Andrew C, Miyoung C, Paul G, Steven K, Aristides P (2015) Using big data to understand the human condition: the Kavli HUMAN project. Big Data 3(3):173-188

73. Rudder C (2014) Dataclysm: who we are (when we think no one's looking). Random House, New York, pp 127-136

74. San Pedro J, Proserpio D, Oliver N (2015) MobiScore: towards universal credit scoring from mobile phone data. In: Proceedings of international conference on user modeling, adaptation and personalization, pp 195-207

75. Singh VK, Bozkaya B, Pentland A (2015) Money walks: implicit mobility behavior and financial well-being. PLoS ONE 10(8):e0136628

76. Barlacchi G, De Nadai M, Larcher R, Casella A, Chitic C, Torrisi G, Antonelli F, Vespignani A, Pentland A, Lepri B (2015) A multi-source dataset of urban life in the city of Milan and the Province of Trentino. Sci Data 2:150055 